\title{
Urine NGAL and KIM-1 in children and adolescents with hyperuricemia
}

\author{
Justyna Tomczak • Anna Wasilewska • Robert Milewski
}

Received: 27 February 2013 /Revised: 8 April 2013 /Accepted: 10 April 2013 /Published online: 15 May 2013

(C) The Author(s) 2013. This article is published with open access at Springerlink.com

\begin{abstract}
Background The aim of this study was to test the hypothesis that urine levels of neutrophil gelatinase-associated lipocalin (NGAL) and kidney injury molecule-1 (KIM-1) are enhanced in pediatric patients with hyperuricemia.

Methods The study included 88 children and adolescents (60 males, 28 females) with a median age of 16 (range 11-18.5) years who had been referred to our department to rule out or confirm hypertension. The subjects were divided into two groups: the hyperuricemic (HU) group comprising 59 subjects with hyperuricemia (defined as serum uric acid $>4.8$ and $>5.5 \mathrm{mg} / \mathrm{dl}$ in girls and boys, respectively) and the reference group comprising 29 patients with normouricemia. Urine NGAL and KIM-1 levels were evaluated using a commercially available kit.

Results Concentrations of the examined biomarkers [urine NGAL, NGAL/creatinine (cr.) ratio, urine KIM-1, KIM$1 /$ cr. ratio] were increased in the HU group compared with the reference group $(p<0.01)$. There were positive correlations between the serum uric acid and urine NGAL/cr. ratio $(R=0.67, p<0.001)$ and the urine KIM- $1 /$ cr. ratio $(R=0.36$, $p<0.001)$. In the multiple regression models, serum uric acid, systolic blood pressure and cholesterol accounted for more than $49 \%$ of the variation in the NGAL/cr. ratio $(R=$ $0.702, p<0.001)$. In the second model, serum uric acid, gender, age and systolic blood pressure accounted for more than $36 \%$ of the variation in the KIM- $1 / \mathrm{cr}$. ratio $(R=0.604$, $p<0.001$ ).
\end{abstract}

J. Tomczak · A. Wasilewska $(\bowtie)$

Department of Paediatrics and Nephrology, Medical

University of Bialystok, ul. Waszyngtona 17,

15-274 Białystok, Poland

e-mail: annwasil@interia.pl

R. Milewski

Department of Statistics and Medical Informatics, Medical

University of Bialystok, Bialystok, Poland
Conclusion We demonstrated that male, obese, hypertensive adolescents with hyperuricemia have higher urine NGAL and KIM-1 levels relative to a reference group with normouricemia.

Keywords Hyperuricemia · Urine NGAL · Urine KIM-1 · Hypertensive children and adolescents · Chronic kidney disease

\section{Introduction}

The association between uric acid and chronic kidney disease (CKD) has been known since the 1890s [1]. There is growing evidence that CKD is associated with hyperuricemia, but debate remains ongoing on whether renal impairment is due to a direct nephrotoxic effect of uric acid or due to the conditions caused by hyperuricemia. Recent experimental studies have demonstrated that hyperuricemia causes hypertension, afferent arteriolopathy, glomerular hypertrophy and increased glomerular pressure, but also tubulointerstitial damage [2-5]. Most studies have focused on uric acid-induced endothelial dysfunction, oxidative stress and inflammation in the kidney. The direct effects of uric acid on tubular cells have not been studied in detail, although the recent study by Ryu et al. [5] showed that uric acid, at physiological concentrations, could induce phenotypic transition of renal tubular cells via both a decreased synthesis and an enhanced degradation of Ecadherin, thereby initiating epithelial-mesenchymal transition of renal tubules and interstitial fibrosis.

Most of the recent evidence for a direct pathogenic effect of hyperuricemia on the kidneys comes from animal studies. However, studies in humans have been demonstrated that hyperuricemia is associated with a decline in kidney function and that allopurinol might slow this decline $[6,7]$. In one prospective study, patients with asymptomatic hyperuricemia 
treated with allopurinol showed significant improvement in glomerular filtration rate (GFR) after 3 months of the therapy [8]. Siu et al. reported that among patients with CKD treated with allopurinol, $16 \%$ progressed to end-stage renal disease compared to $46 \%$ in the control group [9].

Tubulointerstitial damage is characterized by inflammatory cell infiltrates, loss of peritubular capillaries, tubular atrophy and interstitial fibrosis. Tubular cells are important players in the initiation and progression of renal damage. Several tubular damage markers have recently been identified, and increased levels of these markers indicate tubular damage or acute kidney injury after various nephrotoxic insults, such as ischemia during cardiac surgery, sepsis and the administration of contrast medium [10]. However, few clinical studies have been performed in patients with CKD. In addition, to the best of our knowledge the urinary levels of tubular damage biomarkers in patients with hyperuricemia have not been studied.

Neutrophil gelatinase-associated lipocalin (NGAL) is a promising emerging biomarker for early detection of kidney injury. It is a member of the lipocalin family that is expressed at low levels in several human tissues and rapidly released from renal tubular cells in response to various insults to the kidney $[11,12]$.

Another very well characterized urinary biomarker in both experimental animals and humans with renal disease is kidney injury molecule (KIM-1), which was isolated in 1998 by Ichimura et al. [13]. KIM-1 is a transmembrane type 1 glycoprotein expressed at very low levels in the normal kidney. It is markedly upregulated in proximal tubules following renal ischemic or toxic injury in humans and experimental animals $[13,14]$. Cherian et al. found that KIM-1 is a sensitive marker of early injury to tubular cells [15]. The results of experimental and clinical studies show that elevated urinary KIM-1 levels are strongly related to tubular KIM-1 expression, indicating that KIM-1 is a very promising biomarker for the presence of tubule-interstitial pathology [16].

Whether tubular injury plays a role in mild hyperuricemia remains unclear. Therefore, the aim of this study was to test the hypothesis that urinary levels of NGAL and KIM-1 are enhanced in pediatric patients with hyperuricemia.

\section{Patients and methods}

Informed consent was obtained from parents of all participants and children older than 16 years of age. The protocol was approved by the Bioethics Committee of the Medical University of Białystok in accordance with the Declaration of Helsinki.

The study included 88 children and adolescents $(60$ males, 28 females) aged $11-18$ years who were referred to our unit (Department of Pediatrics and Nephrology, The Medical University of Białystok, Poland) between May 2010 and September 2011 in order to confirm or rule out hypertension. The majority of subjects were referred to our department by general practitioners who had determined that the patient had elevated causal blood pressure (BP). The subjects were divided into two groups: a hyperuricemic (HU) and normouricemic (reference) group, respectively. The HU group comprised 59 children and adolescents (49 males, 10 females) with hyperuricemia, defined as serum uric acid $>4.8 \mathrm{mg} / \mathrm{dl}$ in girls and serum uric acid $>5.5 \mathrm{mg} / \mathrm{dl}$ in boys [17], confirmed primary hypertension or white-coat hypertension. The reference group comprised 29 teenagers (11 males, 18 females) with normouricemia and in whom at the moment of examination hypertension was excluded on the basis of ambulatory blood pressure monitoring (ABPM; mean systolic and diastolic daytime and nighttime BP levels of $<90$ th percentile for age, sex and height and load systolic and diastolic BP of $<25 \%$ ). The participants in the reference group had been full-term babies, with a normal birth weight, and were not receiving any medication at the time of the study. Family history of adolescents qualifying for this group did not reveal gout or hypertension.

Inclusion criteria for the study for all participants were an age of 11-18 years, normal clinical examination, normal renal function and patients who were not receiving antihypertensive or uric acid-reducing therapy. Exclusion criteria for entry into the study were heart failure, diabetes mellitus, renal or hepatic dysfunction, hematological disease, thrombocytopenia, systemic inflammatory conditions and autoimmune diseases.

For all subjects, careful clinical histories were taken and physical examinations were performed. Body weight and height were measured using a balance beam scale and pediatric wall-mounted stadiometer, respectively, and body mass index (BMI) was calculated as weight (in kilograms) divided by the square of height (meters squared). BMI $Z$-scores reflecting the standard deviation score (SDS) for the ageand gender-appropriate BMI distribution were calculated using the formula: BMI $Z$-scores $=(50$ percentile for age + gender $) / 1 / 2 \times(50$ percentile -3 percentile for age and gender $)$.

After $12 \mathrm{~h}$ of night fasting, between 7 and 8 a.m., urine and blood samples were taken for the measurement of the urine concentration of NGAL, KIM-1, creatinine (cr.) and albuminuria, plasma $\mathrm{C}$-reactive protein level and serum creatinine, urea and uric acid levels, determination of the lipid profile, and morphological profile of the peripheral blood. Serum creatinine was determined by Jaffe reaction, and uric acid was measured using the Hitachi apparatus (Hitachi, Tokyo, Japan). The morphological characteristics of the peripheral blood were assessed using the Beckman Coulter MAXM AL flow cytometer (Beckman Coulter, Fullerton, CA). Serum cholesterol, high-density lipid (HDL)-cholesterol and triglycerides (TG) were determined 
by the enzymatic method using the Hitachi 912 chemistry analyzer (Roche Diagnostics, Indianapolis, IN).

The 24-h urine albumin excretion rate (UAER) was analyzed using a radioimmunoassay method. Albuminuria was considered as 24-h UAER $>30 \mathrm{mg} / 24 \mathrm{~h}$. Micro- and macroalbuminuria were defined as 24 -h UAER values of 30-300 and $>300 \mathrm{mg} / 24 \mathrm{~h}$, respectively. The estimated glomerular filtration rate (eGFR) was calculated from the Schwartz formula: eGFR $=0.413 \times$ growth $(\mathrm{cm}) /$ serum cr. $(\mathrm{mg} / \mathrm{dl})$.

The concentration of urinary NGAL and KIM-1 was measured using a commercially available enzyme-linked immunosorbent assay (ELISA) kit according to the manufacturer's instructions. All specimens were diluted to obtain the concentration for the optimal density according to the instructions for the ELISA kit. Urine NGAL and urine KIM1 levels were expressed in nanograms per milliliter. Urine creatinine concentration was used to normalize the NGAL and KIM-1 measurements to account for the influence of urinary dilution on its concentration. Finally, urine NGAL and KIM-1 levels were expressed as the NGAL/cr. and $\mathrm{KIM}-1 / \mathrm{cr}$. ratio, respectively, in nanograms per milligram of creatinine.

ABPM was performed using Spacelabs Healthcare CardioNavigator (Spacelabs Healthcare, Issaquah, WA). The monitors were programmed to measure BP every 15 min during daytime (0800-2200 hours) and every 30 min during nighttime (2200-08.00 hours); however, these periods were corrected according to each patient's diary. Hypertension on the basis of ABPM was defined as mean systolic or diastolic daytime or nighttime BP levels that were $\geq 95$ th percentile.

\section{Statistical analysis}

All continuous variables were tested for normal distribution using the Kolmogorov-Smirnov test with the Lillefor's correction and the Shapiro-Wilk test. As most of the studied parameters were not normally distributed, descriptive statistics were calculated as the median with the interquartile range. The Mann-Whitney $U$ test was used to compare continuous variables, and the chi-square $\left(\chi^{2}\right)$ test with Yates' correction was used to compare categorical variables between two groups. Correlations between uric acid, the NGAL/cr. ratio, the KIM-1/cr. ratio and other studied variables were assessed using Spearman's correlation test. Two multiple linear regression models were created that included the NGAL/cr. ratio and KIM-1/cr. ratio as dependent variables. Statistical significance was determined at $p<0.05$. All calculations were made using the Statistica ver. 10.0 program (StatSoft Inc. Tulsa, OK) and the Stata/IC ver. 12.1 program (StataCorp LP, College Station, TX).

\section{Results}

The clinical and biochemical characteristics of the study participants are listed in Table 1. The age of the HU patients did not differ from that of the healthy controls $(p=0.21)$. Males were more frequently affected with hyperuricemia than females. There were more males than females in the HU group [49 (83.05\%) vs. 10 (16.95\%), respectively], whereas more females $(n=18,62.07 \%)$ than males $(n=11$, $37.93 \%)$ were found in the reference group $\left(\chi^{2}\right.$ test 18.24: $p<0.01)$.

The body height, body weight and BMI of HU adolescents were higher than those of adolescents in the reference group $(p<0.001)$, which can be partially explained by the difference in number of males and females in each group. However, the median BMI Z-score in the HU group was 2.12 (IQR 0.90-3.87) and higher than that found for the reference group $(p<0.001)$. Thirty-six subjects $(61.01 \%)$ from the HU group were classified as overweight or obese. Compared to the reference group, the HU patients had increased serum creatinine $(p<0.001)$ and urea levels $(p<0.01)$ and decreased eGFR $(p<0.001)$. HU subjects also had a higher cholesterol level and 24-h UAER than those in the reference group $(p<0.05$ and $p<0.001$, respectively) (Fig. 1). We found significant differences in the urine NGAL and urine NGAL/cr. ratio, and in the urine KIM-1 and KIM-1/cr. ratio between HU patients and the reference group. The median urine NGAL concentration (in $\mathrm{ng} / \mathrm{ml}$ ) and NGAL/cr. ratio were 5.7- and 7.6-fold higher in $\mathrm{HU}$ patients than in the reference group. There was a statistically significant correlation $(p<0.001, R=0.56)$ between urine $\mathrm{KIM}-1 / \mathrm{cr}$. and urine NGAL/cr. levels in the HU group.

Single regression analyses revealed that the serum uric acid level was positively correlated with both the $\mathrm{NGAL} / \mathrm{cr}$. and KIM-1/cr. ratios $(R=0.67, p<0.001$ and $R=0.37, p<0.001$, respectively). The urine NGAL/cr. ratio was also positively correlated with body weight $(R=0.32, p=0.002)$, BMI $Z$-score $(R=0.37, p<0.001)$, serum creatinine level $(R=0.28, p=0.007)$, urea $(R=0.25, \mathrm{p}=0.02)$, total cholesterol level $(R=0.27$, $p=0.01)$, 24-h UAER $(R=0.39, p<0.001)$, systolic BP $(R=0.25, p=0.02)$ and diastolic $\mathrm{BP}(R=0.29, p=0.008)$ and negatively correlated with eGFR $(R=-0.26, p=0.01)$. The urine KIM-1/cr. ratio was correlated positively with body weight $(R=$ $0.27, p=0.01)$, BMI $Z$-score $(R=0.3, p=0.004)$ and serum creatinine levels $(R=0.31, p=0.003)$ and negatively correlated with eGFR $(R=-0.29, p<0.01)$.

The factors that were found to have a significant correlation with the urine $\mathrm{NGAL} / \mathrm{cr}$. ratio and $\mathrm{KIM}-1 / \mathrm{cr}$. ratio in the single regression analyses were used as explanatory variables to create the multiple regression models. To reduce the impact of multicollinearity we first removed a number of correlated variables and then eliminated a few more non- 
Table 1 Clinical and biochemical characteristics of the group with hyperuricemia and the reference group (normouricemia)

\begin{tabular}{|c|c|c|c|}
\hline Clinical and biochemical parameters & $\begin{array}{l}\text { HU }(N=59) \\
\text { Median }(\mathrm{Q} 1 ; \mathrm{Q} 3) \text { or proportion }\end{array}$ & $\mathrm{R}(N=29)$ & $p$ value \\
\hline Age (years) & $16(15 ; 17)$ & $16(15 ; 17)$ & NS \\
\hline Sex (male/female), $n$ (\% male) & $49 / 10(83.05 \%)$ & $11 / 18(37.93 \%)$ & $<0.001$ \\
\hline$Z$-score BMI & $2.12(0.90 ; 3.87)$ & $-0.35(-1.14 ; 0.46)$ & $<0.001$ \\
\hline Serum uric acid (mg/dl) & $6.7(6.23 ; 7.31)$ & $4.47(3.8 ; 4.82)$ & $<0.001$ \\
\hline Serum creatinine $(\mathrm{mg} / \mathrm{dl})$ & $0.79(0.69 ; 0.91)$ & $0.56(0.47 ; 0.68)$ & $<0.001$ \\
\hline Urea (mg/dl) & $25.5(22 ; 29)$ & $22(19 ; 24)$ & 0.002 \\
\hline Serum cholesterol (mg/dl) & $183(144 ; 201)$ & $146(139 ; 174)$ & 0.02 \\
\hline TG/HDL ratio & $2.52(1.59 ; 3.68)$ & $1.64(1.22 ; 2.43)$ & NS \\
\hline eGFR (ml/min/1.73 m²) & $90.10(80.55 ; 103.57)$ & $118.64(106.2 ; 148.34)$ & $<0.001$ \\
\hline 24-h urine albumin excretion rate (UAER) $\mathrm{mg} / 24-\mathrm{h}$ & $44.3(8.64 ; 56.3)$ & $9.5(4.75 ; 14.3)$ & $<0.001$ \\
\hline $24 \mathrm{SBP}(\mathrm{mmHg})$ & $132(122.5 ; 137)$ & $118(112 ; 122.5)$ & $<0.001$ \\
\hline $24 \mathrm{DBP}(\mathrm{mmHg})$ & $71(66.5 ; 75)$ & $66(61.5 ; 70)$ & $<0.001$ \\
\hline uNGAL (ng/ml) & $8.54(3.04 ; 18.55)$ & $1.49(0.81 ; 3.24)$ & $<0.001$ \\
\hline NGAL/cr. (ng/mg cr.) & $8.68(2.08 ; 23.51)$ & $1.14(0.63 ; 2,58)$ & $<0.001$ \\
\hline uKIM-1 (ng/ml) & $1.3(0,99 ; 2.1)$ & $0.85(0.38 ; 1.34)$ & $<0.001$ \\
\hline KIM-1/cr. (ng/mg cr.) & $1.26(0.76 ; 1.87)$ & $0.75(0.46 ; 1.14)$ & $<0.001$ \\
\hline
\end{tabular}

Data in table are presented as the median with the interquartile range $\left(\mathrm{IQR} ; \mathrm{Q}_{1} ; \mathrm{Q}_{3}\right)$ given in parenthesis, unless stated otherwise

HU, Hyperuricemic group; R, reference group (normouricemia); BMI body mass index; TG, triglycerides; HDL, high-density lipoprotein; eGFR, estimated glomerular filtration rate; SBP, systolic blood pressure; DBP, diastolic blood pressure; uNGAL, urine neutrophil gelatinase-associated lipocalin; UKIM-1, urine kidney molecule-1; NS, not significant
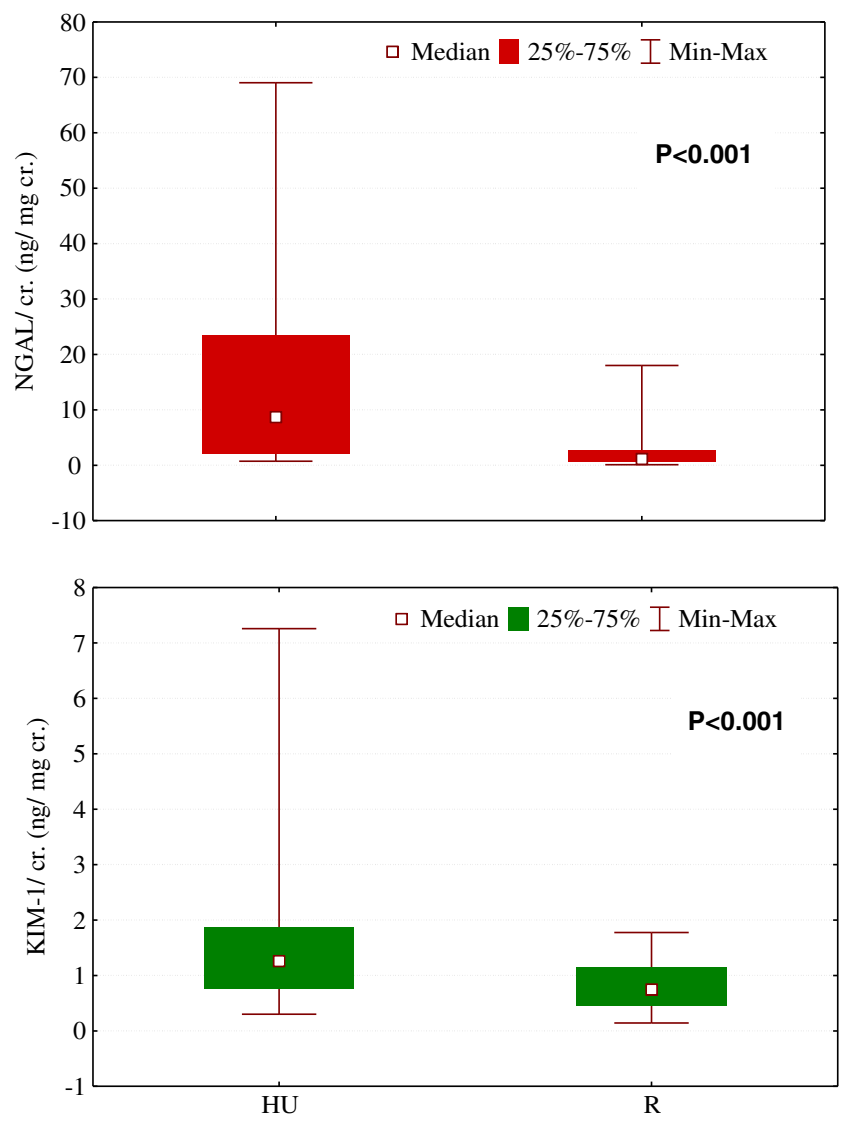

Fig. 1 Comparison of the urine neutrophil gelatinase-associated lipocalin/ creatinine (NGAL/cr.) ratio and the kidney injury molecule-1 (KIM-1)/cr. ratio between patients with hyperuricemia and the reference group significant (in the model) variables. This procedure resulted in the creation of the two models shown in Tables 2 and Table 3, respectively. In the first model (Table 2), the remaining three parameters (serum uric acid, systolic BP and cholesterol level) accounted for more than $49 \%$ of the variation in the NGAL/cr. ratio $(R=0.702, p<0.001)$. In the second model (Table 3 ), the remaining four parameters (serum uric acid, gender, age and systolic BP) accounted for more than $36 \%$ of the variation in the $\mathrm{KIM}-1 / \mathrm{cr}$. ratio ( $R=0.604, p<0.001)$. It is interesting to note that male gender correlated negatively with the KIM- $1 / \mathrm{cr}$. ratio in this model.

\section{Discussion}

Our results demonstrate that male, obese, hypertensive adolescents with hyperuricemia have higher urine NGAL and KIM-1 levels than adolescents with normouricemia (the reference group). Another important finding was the positive correlations between serum uric acid and the urine NGAL/cr. and KIM-1/cr. ratios. Additionally, both urine biomarkers correlated with parameters of renal function. This correlation is in accordance with the results reported by other authors who confirmed this relationship in immunoglobulin A (IgA) nephropathy, diabetic nephropathy and autosomal dominant polycystic kidney disease [18-20]. 
Table 2 Multiple linear regression analysis of the NGAL/creatinine ratio

\begin{tabular}{lllll}
\hline Variables & Coefficient & Standard error & $95 \%$ Confidence interval & $p$ value \\
\hline Serum uric acid & 7.24 & 1.014 & $(5.22 ; 9.27)$ & $<0.001$ \\
Systolic blood pressure & -0.137 & 0.0478 & $(-0.233 ;-0.042)$ & 0.005 \\
Cholesterol & 0.0996 & 0.0399 & $(0.0201 ; 0.1792)$ & 0.02 \\
\hline
\end{tabular}

However, the results of our study raise the challenging question of the "chicken and egg" dilemma with regard to uric acid and renal adverse outcomes. It is possible that the uric acid is causing, or contributing to, subclinical kidney disease.

The epidemic of metabolic syndrome, a major public health problem, correlates with an excessive intake of fructose [21, 22]. Recent studies have demonstrated that urinary uric acid excretion is remarkably reduced in fructose-fed rats, resulting in hyperuricemia which in turn plays a pathogenic role in renal dysfunction and metabolic syndrome $[22,23]$. A large and growing body of evidence suggests that hyperuricemia might be a risk factor for renal dysfunction [24]. Hu et al. [25] demonstrated that in fructose-fed rats, not only was there a reduction in urine uric acid and creatinine excretion, but there was also a significant elevation of serum creatinine and blood urea nitrogen levels, indicating renal dysfunction.

The molecular mechanism for fructose-induced hyperuricemia was proposed by Le et al. [26]. These authors demonstrated that SLC22A, one of classic polyspecific urate transporters in the proximal kidney tubule which take part in the reabsorption and secretion of renal uric acid, plays a pivotal role in renal dysfunction. The organic cation transporters OCT1 (SLC22A1) and OCT2 (SLC22A2) in the proximal renal tubules cooperate with each other in the excretion of organic cations from the kidneys. Decreased expression level of rOCT2 in the rat kidney was shown in ischemia- and reperfusion-induced acute kidney injury [27] and in hyperuricemia [28]. The reduction of renal expression of other transporters was also reported in streptozotocin-induced diabetic rats [29] and nephrotoxicity [30].

The mechanism of HU nephropathy is still a matter of debate. Previous studies have demonstrated uric acidinduced endothelial dysfunction and local inflammation in the kidney as major mechanisms of renal disease $[2,7,24$, $31,32]$. However, the novel finding of Ryu et al. [5] strongly suggest that uric acid also has direct effects on renal tubules. In this study, the authors observed phenotypic transition of the renal tubules before the development of renal dysfunction and significant tubulointerstitial fibrosis in hyperuricemic rats, which was ameliorated by uric acidlowering therapy. The study by Han et al. [33] showed uric acid-induced inhibition of tubular cell proliferation in vitro. Another study demonstrated an upregulation of ERK, BAX and $\alpha$-SMA in HK-2 cells exposed to uric acid using SILAC coupled to liquid chromatography-mass spectrometry [34].

In our study we confirmed an increased urine level of tubule damage markers in male adolescents with hyperuricemia. It is worth noting that the urine NGAL/cr. ratio in patients with hyperuricemia was about sevenfold higher than that in the normouricemic subjects in the reference group, while the urine KIM-1/cr. ratio exceeded the values of reference group by only twofold. It is difficult to explain this result because we found a statistically significant correlation between the urine NGAL/cr. and KIM-1/cr. ratios. One possible explanation might be that the urine KIM-1/cr. ratio correlates with gender and is lower in males; in our study, there was a preponderance of males in the HU group.

A review of the literature found no data on the association between serum uric acid and the urine $\mathrm{NGAL} / \mathrm{cr}$. or KIM-1/cr. ratios. Urine NGAL has been studied extensively in preclinical and clinical models and has been found to be a potential biomarker of a number of pathologic conditions, including acute kidney injury, diabetic nephropathy, IgA nephropathy, contrast nephropathy, and a useful tool in disease monitoring in other renal diseases, such as delayed graft function, lupus nephritis, IgA nephropathy and polycystic disease [20, 35-37].

In recent years, there has been an increasing amount of data published on role of KIM-1 in kidney injury. KIM-1 appears to play a role in the pathogenesis of tubular cell damage and repair in experimental and human kidney disease. It has been found to be upregulated after nephrotoxicant-induced renal injury [38] and in the murine model of polycystic kidney
Table 3 Multiple linear regression analysis of the KIM-1/creatinine ratio

\begin{tabular}{lllll}
\hline Variables & Coefficient & Standard error & $95 \%$ Confidence interval & $p$ value \\
\hline Serum uric acid & 0.464 & 0.0799 & $(0.305 ; 0.624)$ & $<0.001$ \\
Gender & -0.729 & 0.242 & $(-1.212 ;-0.246)$ & 0.004 \\
Age & 0.119 & 0.0609 & $(-0.0021 ; 0.2405)$ & 0.05 \\
Systolic blood pressure & -0.0103 & 0.0039 & $(-0.018 ;-0.0025)$ & 0.01 \\
\hline
\end{tabular}


disease [39] and human renal carcinoma [40]. Vaidya et al. [41] obtained interesting results which demonstrated that KIM-1 was increased 1 day after ischemia-reperfusion injury in rats, before a rise in proteinuria or plasma creatinine could be observed, indicating that KIM-1 is a very early, specific biomarker of acute kidney injury. As already mentioned, very little information is currently available in the literature on the influence of uric acid on urine KIM-1 levels. Nepal et al. [42] reported one case of acute urate nephropathy and one case of chronic urate nephropathy in two pediatric patients. These authors demonstrated the upregulation of KIM-1 in proximal tubular cells, which implicates a role for KIM-1 in proximal tubular injury in urate nephropathy. This observation was confirmed in our study. It is also worth mentioning that some hyperuricemic patients had first or second degree CKD based on a decreased eGFR, elevated levels of serum creatinine and albuminuria. We also found statistically significant correlations between both NGAL and KIM-1 and parameters or renal function. These results lead us to consider that elevations in NGAL and KIM-1 levels may reflect the degree of CKD due to hyperuricemia. However, this notion must be confirmed in larger group of patients.

Another point which should be discussed is the positive correlation between the NGAL/cr. ratio and components of metabolic syndrome. In our study, most of the HU patients $(67.79 \%)$ were hypertensive, with $61.01 \%$ being overweight or obese. Our finding of a correlation between NGAL and BP values is in agreement with those reported by Eirin et al. [43] who showed that urinary levels of NGAL were elevated in both essential hypertension and renovascular hypertension. It seems possible that these results were due to ongoing kidney and systemic inflammation and ischemia in hypertensive patients. These findings further support the notion of Blumczynski et al. [44] who considered that in children with primary hypertension, increased serum and urine NGAL may reflect kidney injury earlier than typical markers of hypertensive nephropathy. Recent evidence suggests that NGAL may also be involved in chronic vascular remodeling during the development of atherosclerosis [45]. In our study we found a significant correlation between NGAL and serum cholesterol level, but not with TG level and the TG/HDL ratio (which is considered to be a marker of atherosclerosis). In reviewing the literature, we found no data on the association between urine NGAL and BMI or obesity; therefore, further research should be conducted to investigate this relationship.

We also found a positive correlation between the urine $\mathrm{KIM}-1 / \mathrm{cr}$. ratio and the BMI $Z$-score. However, we were unable to obtain any data on this relationship in the literature.

The findings of this study suggest that urine NGAL rather than urine KIM-1 may serve as an early marker of tubular damage due to hyperuricemia. However, this research may only serve as a base for future studies.
Finally, a number of important limitations to this study need to be considered. First, this study is limited by its relatively small study population and cross-sectional nature. Secondly, the reference group was not a group of healthy teenagers, but a group of patients with normouricemia, in whom hypertension was not confirmed in 24-h ABPM, so they were considered to be the white coat hypertension group. Thirdly, there were the evident differences between the study groups, with the HU group consisting of predominantly of adolescent males mainly with a high BMI $Z$-score and reference group including relatively more females with white coat hypertension.

Acknowledgments This study was supported by a research grant from the Medical University of Bialystok (No 123-41765 L).

\section{Conflict of interest None.}

Open Access This article is distributed under the terms of the Creative Commons Attribution License which permits any use, distribution, and reproduction in any medium, provided the original author(s) and the source are credited.

\section{References}

1. Feig DI (2009) Uric acid: a novel mediator and marker of risk in chronic kidney disease? Curr Opin Nephrol Hypertens 18:526-530

2. Mazzali M, Kanellis J, Han L, Feng L, Xia YY, Chen Q, Kang DH, Gordon KL, Watanabe S, Nakagawa T, Lan HY, Johnson RJ (2002) Hyperuricemia induces a primary renal arteriolopathy in rats by a blood pressure-independent mechanism. Am J Physiol Renal Physiol 282:F991-F997

3. Sánchez-Lozada LG, Tapia E, Santamaría J, Avila-Casado C, Soto $\mathrm{V}$, Nepomuceno T, Rodríguez-Iturbe B, Johnson RJ, HerreraAcosta J (2005) Mild hyperuricemia induces vasoconstriction and maintains glomerular hypertension in normal and remnant kidney rats. Kidney Int 67:237-247

4. Nakagawa T, Mazzali M, Kang DH, Kanellis J, Watanabe S, Sanchez-Lozada LG, Rodriguez-Iturbe B, Herrera-Acosta J, Johnson RJ (2003) Hyperuricemia causes glomerular hypertrophy in the rat. Am J Nephrol 23:2-7

5. Ryu ES, Kim MJ, Shin HS, Jang YH, Choi HS, Jo I, Johnson RJ, Kang DH (2013) Uric acid- induced phenotypic transition of renal tubular cells as a novel mechanism of chronic kidney disease. Am J Physiol Renal Physiol 304:F471-F480

6. Ji B, Zhang S, Gong L, Wang Z, Ren W, Li Q, Li R (2013) The risk factors of mild decline in estimated glomerular filtration rate in a community-based polulation. Clin Biochem. doi: 10.1016/ j.clinbiochem.2013.01.011

7. Feig DI, Kang DH, Johnson RJ (2008) Uric acid and cardiovascular risk. N Engl J Med 359:1811-1821

8. Kanbay M, Ozkara A, Selcoki Y, Isik B, Turgut F, Bavbek N, Uz E, Akcay A, Yigitoglu R, Covic A (2007) Effect of treatment of hyperuricemia with allopurinol on blood pressure, creatinine clearence, and proteinuria in patients with normal renal functions. Int Urol Nephrol 39:1227-1233

9. Siu YP, Leung KT, Tong MK, Kwan TH (2006) Use of allopurinol in slowing the progression of renal disease through its ability to lower serum uric acid level. Am J Kidney Dis 47:51-59 
10. Bagshaw SM, Bellomo R (2007) Early diagnosis of acute kidney injury. Curr Opin Crit Care 13:638-644

11. Venge P, Carlson M, Fredens K, Garcia R (1999) The 40 kDprotein. A new protein isolated from the secondary granules of human neutrophils: Joint International Conference on Leukocyte Biology. J Leukocyte Biol 1:28

12. Allen RA, Erickson RW, Jesaitis AJ (1989) Identification of a human neutrophil protein of $\mathrm{Mr} 24000$ that binds $\mathrm{N}$-formyl peptides: co-sedimentation with specific granules. Biochim Biophys Acta 991:123-133

13. Ichimura T, Bonventre JV, Bailly V, Wei H, Hession CA, Cate RL, Sanicola M (1998) Kidney injury molecule-1 (KIM-1), a putative epithelial cell adhesion molecule containing a novel immunoglobulin domain, is up-regulated in renal cells after injury. J Biol Chem 273:4135-4142

14. Han WK, Bailly V, Abichandani R, Thadhani R, Bonventre JV (2002) Kidney Injury Molecule-1 (KIM-1): a novel biomarker for human renal proximal tubule injury. Kidney Int 62:237-244

15. Cherian S, Crompton $\mathrm{CH}$ (2005) Partial hypoxanthine-guanine phosphoribosyltransferase deficiency presenting as acute renal failure. Pediatr Nephrol 20:1811-1813

16. Lock EA (2010) Sensitive and early markers of renal injury: where are we and what is the way forward? Toxicol Sci 116:1-4

17. Feig DI, Johnson RJ (2003) Hyperuricemia in childhood primary hypertension. Hypertension 42:247-252

18. Xu PC, Zhang JJ, Chen M, Lv JC, Liu G, Zou WZ, Zhang H, Zhao MH (2011) Urinary kidney injury molecule-1 in patients with IgA nephropathy is closely associated with disease severity. Nephrol Dial Transplant 26:3229-3236

19. Yang YH, He XJ, Chen SR, Wang L, Li EM, Xu LY (2009) Changes of serum and urine neutrophil gelatinase-associated lipocalin in type2 diabetic patients with nephropathy: one year observational followup study. Endocrine 36:45-51

20. Bolignano D, Coppolino G, Campo S, Aloisi C, Nicocia G, Frisina N, Buemi M (2007) Neutrophil gelatinase-associated lipocalin in patients with autosomal-dominant polycystic kidney disease. Am J Nephrol 27:373-378

21. Miatello R, Vázquez M, Renna N, Cruzado M, Zumino AP, Risler N (2005) Chronic administration of resveratrol prevents biochemical cardiovascula changes in fructose-fed rats. Am J Hypertens 18:864-870

22. Nakagawa T, Hu H, Zharikov S, Tuttle KR, Short RA, Glushakova O, Ouyang X, Feig DI, Block ER, Herrera-Acosta J, Patel JM, Johnson RJ (2006) A causal role for uric acid in fructose-induced metabolic syndrome. Am J Physiol Renal Physiol 290:F625-F631

23. Gersch MS, Mu W, Cirillo P, Reungjui S, Zhang L, Roncal C, Austin YY, Johnson RJ, Nakagawa T (2007) Fructose, but not dextrose, accelerates the progression of chronic kidney disease. Am J Physiol Renal Physiol 293:F1256-F1261

24. Kang DH, Nakagawa T, Feng L, Watanabe S, Han L, Mazzali M, Truong L, Harris R, Johnson RJ (2002) A role for uric acid in the progression of renal disease. J Am Soc Nephrol 13:2888-2897

25. Hu QH, Wang C, Li JM, Zhang DM, Kong LD (2009) Allopurinol, rutin, and quercetin attenuate hyperuricemia and renal dysfunction in rats induced by fructose intake: renal organic ion transporter involvement. Am J Physiol Renal Physiol 297:F1080-F1091

26. Le MT, Shafiu M, Mu W, Johnson RJ (2008) SLC2A9-A fructose transporter identified as a novel uric acid transporter. Nephrol Dial Transplant 23:2746-2749

27. Matsuzaki T, Watanabe H, Yoshitome K, Morisaki T, Hamada A, Nonoguchi H, Kohda Y, Tomita K, Inui K, Saito H (2008) Altered pharmacokinetics of cationic drugs caused by down-regulation of renal rat organic cation transporter 2 (Slc22a2) and rat multidrug and toxin extrusion 1 (Slc47a1) in ischemia/reperfusion-induced acute kidney injury. Drug Metab Dispos 36:649-654

28. Habu Y, Yano I, Okuda M, Fukatsu A, Inui K (2005) Restored expression and activity of organic ion transporters rOAT1, rOAT3 and rOCT2 after hyperuricemia in the rat kidney. Biochem Pharmacol 69:993-999

29. Grover B, Buckley D, Buckley AR, Cacini W (2004) Reduced expression of organic cation transporters rOCT1 and rOCT2 in experimental diabetes. J Pharmacol Exp Ther 308:949-956

30. Rizwan AN, Burckhardt G (2007) Organic anion transporters of the SLC22 family: biopharmaceutical, physiological, and pathological roles. Pharm Res 24:450-470

31. Corry DB, Eslami P, Yamamoto K, Nyby MD, Makino H, Tuck ML (2008) Uric acid stimulates vascular smooth muscle cell proliferation and oxidative stress via the vascular renin-angiotensin system. J Hypertens 26:269-275

32. Yu MA, Sánchez-Lozada LG, Johnson RJ, Kang DH (2010) Oxidative stress with an activation of the renin-angiotensin system in human vascular endothelial cells as a novel mechanism of uric acid-induced endothelial dysfunction. J Hypertens 28:1234-1242

33. Han HJ, Lim MJ, Lee YJ, Lee JH, Yang IS, Taub M (2007) Uric acid inhibits renal proximal tubule cell proliferation via at least two signaling pathways involving PKC, MAPK, cPLA2, and NFkappaB. Am J Physiol Renal Physiol 292:F373-F381

34. Quan H, Peng X, Liu S, Bo F, Yang L, Huang Z, Li H, Chen X, Di W (2011) Differentially expressed protein profile of renal tubule cell stimulated by elevated uric acid using SILAC coupled to LCMS. Cell Physiol Biochem 27:91-98

35. Ding H, He Y, Li K, Yang J, Li X, Lu R, Gao W (2007) Urinary neutrophil gelatinase- associated lipocalin (NGAL)) is an early biomarker for renal tubulointerstitial injury in IgA nephropathy. Clin Immunol 123:227-234

36. Brunner HI, Mueller M, Rutherford C, Passo MH, Witte D, Grom A, Mishra J, Devarajan P (2006) Urinary neutrophil gelatinaseassociated lipocalin as a biomarker of nephritis in childhood-onset systemic lupus erythematosus. Arthritis Rheum 54:2577-2584

37. Wasilewska A, Taranta-Janusz K, Dębek W, Zoch-Zwierz W, Kuroczycka-Saniutycz E (2011) KIM-1 and NGAL: new markers of obstructive nephropathy. Pediatr Nephrol 26:579-586

38. Ichimura T, Hung CC, Yang SA, Stevens JL, Bonventre JV (2004) Kidney injury molecule-1: a tissue and urinary biomarker for nephrotoxicant-induced renal injury. Am J Physiol Renal Physiol 286:F552-F563

39. Kuehn EW, Park KM, Somlo S, Bonventre JV (2002) Kidney injury molecule-1 expression in murine polycystic kidney disease. Am J Physiol Renal Physiol 283:F1326-F1336

40. Han WK, Alinani A, Wu CL, Michaelson D, Loda M, McGovern FJ, Thadhani R, Bonventre JV (2005) Human kidney injury molecule-1 is a tissue and urinary tumor marker of renal cell carcinoma. J Am Soc Nephrol 16:1126-1134

41. Vaidya VS, Ramirez V, Ichimura T, Bobadilla NA, Bonventre JV (2006) Urinary kidney injury molecule-1: a sensitive quantitative biomarker for early detection of kidney tubular injury. Am J Physiol Renal Physiol 290:F517-F529

42. Nepal M, Bock GH, Sehic AM, Schultz MF, Zhang PL (2008) Kidney injury molecule-1 expression identifies proximal tubular injury in urate nephropathy. Ann Clin Lab Sci 38:210-214

43. Eirin A, Gloviczki ML, Tang H, Rule AD, Woollard JR, Lerman A, Textor SC, Lerman LO (2012) Chronic renovascular hypertension is associated with elevated levels of neutrophil gelatinaseassociated lipocalin. Nephrol Dial Transplant 27:4153-4161

44. Blumczynski A, Sołtysiak J, Lipkowska K, Silska M, Poprawska A, Musielak A, Zaniew M, Zachwieja J (2012) Hypertensive nephropathy in children - do we diagnose early enough? Blood Press 21:233-239

45. Hemdahl AL, Gabrielsen A, Zhu C, Eriksson P, Hedin U, Kastrup J, Thorén P, Hansson GK (2006) Expression of neutrophil gelatinase-associated lipocalin in atherosclerosis and myocardial infarction. Arterioscler Thromb Vasc Biol 26:136-142 